\title{
ORIGINAL ARTICLE \\ The effect of arm position and bed adjustment on comfort and pressure under the shoulders in people with tetraplegia: a randomized cross-over study
}

\author{
AM Raab ${ }^{1,2}$, LA Harvey ${ }^{3}$, M Baumberger $^{4}$ and A Frotzler ${ }^{5}$
}

Study design: Randomized, within-in participant cross-over study.

Objective: The purpose of this study was to determine the effect on comfort and pressure of lying with the shoulders and bed in different positions for people with tetraplegia.

Setting: Rehabilitation hospital.

Methods: Twenty people with tetraplegia were tested lying supine with the shoulders and bed in seven different positions. The positions used a combination of three arm and two bed positions. Six of the positions reflected what is commonly recommended in acute spinal cord injury units including a crucifix-type position. The seventh position was selected by participants and reflected their preferred sleeping position. There were five outcomes: general comfort, shoulder comfort, participant choice of preferred position, peak pressure under the shoulders and areal pressure under the shoulders. Pressure was measured using a pressure mapping system and comfort using a visual analogue scale (VAS).

Results: The participants reported significantly higher $(P<0.01)$ general comfort and shoulder comfort in their self-selected position compared with all other positions. There was no statistical difference in peak pressure $(P=0.15)$ or areal pressure $(P=0.08)$ under the shoulders between the seven positions. Most participants indicated that they preferred to lie with their shoulders adducted and internally rotated and the hands either by their sides or on their stomachs.

Conclusion: The position of the shoulders has little effect on pressure but a notable effect on comfort. Participants preferred to sleep with their arms beside their bodies, not with their arms in a crucifix position as commonly advocated.

Spinal Cord (2014) 52, 152-156; doi:10.1038/sc.2013.150; published online 10 December 2013

Keywords: spinal cord injury; tetraplegia; positioning; comfort; pressure

\section{INTRODUCTION}

Shoulder and upper limb complications for people with tetraplegia are common and limit independence and quality of life. ${ }^{1}$ They are associated with pain, spasticity, subluxation, contractures and oedema. ${ }^{2,3}$ It is widely believed that comprehensive shoulder positioning programs are an important strategy for preventing shoulder and upper limb complications, ${ }^{4,5}$ and that positioning programs are particularly important in the early days after injury. Even as far back as the early 1980s, leading clinicians have advocated for the implementation of shoulder positioning programs. ${ }^{6}$ These programs involve people with tetraplegia spending at least a couple of hours each day with their arms placed in specific positions. There is no consensus about the optimal position but the crucifix position in which the shoulders are positioned in $90^{\circ}$ abduction while the patient is supine on the bed, is commonly used and advocated. ${ }^{4-6}$ Positions that involve elevating the arms are also commonly used. Others have argued that positioning programs should be individualized to patients needs ${ }^{7}$ or that the shoulders should be placed in a variety of positions on a rotating basis. ${ }^{8,9}$
The effectiveness of any shoulder positioning programme for people with tetraplegia is yet to be demonstrated within a clinical trial. ${ }^{4,10}$ However, there is a strong rationale and some interim evidence to justify the belief that these types of programs are effective. For example, there is evidence from studies in other populations to indicate that elevation of the upper limbs will minimize oedema. ${ }^{11}$ The evidence about the effectiveness of positioning as a form of stretch for the prevention of contractures is more controversial ${ }^{10,12}$ but nonetheless animal studies indicate that contractures are best prevented by placing muscles and soft tissues in their stretched and elongated positions for at least some part of each day. ${ }^{13}$ This may prevent the loss of sarcomeres in muscles and subsequent loss of joint mobility. ${ }^{14}$ Some argue that if joint mobility can be maintained, then patients are less likely to develop secondary spasticity, subluxation or shoulder pain. ${ }^{15}$

Integral to any discussion about the effectiveness of shoulder positioning programs should be patient comfort and choice. Clearly, these are important considerations. Comfort may also be related to pressure. That is, some positions may place more pressure under the shoulder than others. The purpose therefore of this study was to

${ }^{1}$ Department of Physiotherapy, Swiss Paraplegic Centre, Nottwil, Switzerland; ${ }^{2}$ Bern University of Applied Sciences, Health, Bern, Switzerland; ${ }^{3}$ Rehabilitation Studies Unit, Sydney Medical School/Northern, University of Sydney, Sydney, New South Wales, Australia; ${ }^{4}$ Acute Medicine and Rehabilitation, Swiss Paraplegic Centre, Nottwil, Switzerland and ${ }^{5}$ Clinical Trial Unit, Swiss Paraplegic Centre, Nottwil, Switzerland

Correspondence: AM Raab, Department of Physiotherapy, Swiss Paraplegic Centre, Nottwil 6270, Switzerland.

E-mail: anja.raab@paraplegie.ch

Received 2 August 2013; revised 8 October 2013; accepted 27 October 2013; published online 10 December 2013 
compare seven different positions for comfort and pressure. Six of the positions reflect what is commonly recommended and the seventh position was selected by participants and reflected their preferred position. The hypothesis was that some positions would be more comfortable than others and result in less pressure under the shoulder. It is important to know how shoulder positioning affects comfort and pressure and patients' preferences because clinical guidelines need to take these factors into account when examining the strength of evidence to support specific recommendations.

\section{MATERIALS AND METHODS}

Experimental design

A randomized, within-in participant cross-over study was conducted to determine the effect of seven different shoulder positions on pressure under the shoulders and comfort in people with tetraplegia. Each participant acted as his/her own control. The study was approved by the local ethics committee of Lucerne, Switzerland. We certify that all applicable institutional and governmental regulations concerning the ethical use of human volunteers were followed during the course of this research.

\section{Participants}

Twenty people with tetraplegia were recruited from the inpatient and outpatient programs of the Swiss Paraplegic Centre, Nottwil (SPZ). People were eligible for inclusion if they had a primary diagnosis of spinal cord injury (SCI) (traumatic or non-traumatic), an American Spinal Injury Association Impairment of $\mathrm{A}$ or $\mathrm{B},{ }^{16}$ sustained their injury 2 months prior to the commencement of the study, had a right and left motor level between $\mathrm{C} 4$ to C8 (inclusive), were over 18 years of age, and spoke sufficient German, French, Italian or English to understand the patient information sheet and provide informed consent. Participants were excluded if they had shoulder pain equal to or greater than $4 / 10$ points on the numeric rating scale in the week prior to assessment, were clinically diagnosed with scoliosis, could not tolerate the shoulder positions or if the positions were medically contraindicated (e.g., due to shoulder operations, pressure ulcers or fractures). In addition, participants were excluded if they weighed more than $100 \mathrm{~kg}$, or if they were pregnant.

Prior to testing, the following demographic information was collected: age, gender, height, weight, body mass index and the number of years post injury. Clinical information included the neurological level of injury, the American Spinal Injury Association Impairment classification and a classification of nightly shoulder pain during the past 4 weeks based on the numeric rating scale (NRS).

\section{Testing protocol}

Testing was always done with participants lying in a supine position on a standard foam mattress (DuoNorm Standard SENECTOVIA). Seven different positions were tested on the same day within a 1.5 hour testing session for each participant. A physiotherapist helped participants into each of the seven positions and ensured the participant was comfortable. The order of the positioning for each participant was computer generated and randomized by an independent person. The allocation was concealed from participants and investigators until after all baseline assessments were completed. A participant was considered to have entered the study once allocation was revealed.

The six standardized positioning conditions utilized a combination of three arm and two bed positions (bed flat or top end of the bed inclined $30^{\circ}$; Figure 1). These six positions were selected to reflect those commonly used in clinical practice. The three different arm positions were achieved with the use of wedges. For all participants, the same pillow and the same wedges were used. The narrow end of the wedge (angle of the wedge $10^{\circ}$ ) was positioned under the glenohumeral joint with the shoulders lying flat on the bed. The broader end of the wedge was placed under the hand. Care was taken to ensure that shoulder rotation was achieved at the shoulder (rather than the forearm) and the elbows were always positioned in extension. The seventh position was selected by the participant.

\section{Outcomes}

There were five outcomes: general comfort, shoulder comfort, participant choice of preferred position, peak pressure under the shoulders and areal pressure under the shoulders.

\section{General comfort and shoulder comfort}

General comfort and shoulder comfort were assessed using a VAS $(10 \mathrm{~cm})$ anchored at one end by 'not at all comfortable' and at the other end by 'extremely comfortable'. Participants were required to rate these two aspects of comfort after lying in each of the seven positions. In addition, they were asked to state what they liked about each position.

\section{Preferred position}

Participants were required to select their preferred position in supine.

\section{Peak- and areal pressure under the shoulders}

Peak- and areal pressure under the shoulders were measured using a pressure mapping system (XSENSOR X3 LX100; Calgary, Alberta, Canada) with data analysed using X3-Software. A $50.8 \times 50.8 \mathrm{~cm}$ pressure pad was placed over the mattress and under participants' upper bodies. The pressure pad comprised $16001 \times 1 \mathrm{~cm}$ sensors, which each measured pressure in $\mathrm{mmHg}$ and recorded at a rate of $20 \mathrm{~Hz}$. The system was calibrated by a medical engineer according to the manufacturer's recommendations prior to the commencement of the study. In addition, the test-retest reliability of the device was tested by measuring peak- and areal pressure on two occasions in 15 able-bodied participants. The intraclass correlation coefficient for areal pressure was 0.81 $(P<0.01,95 \%$ CI 0.52 to 0.93$)$ reflecting good reliability and for peak pressure was $0.42(P=0.06,95 \% \mathrm{CI}-0.12$ to 0.77$)$ reflecting poor reliability.

Peak- and areal pressure under the shoulders were derived from the sensors under the shoulder region. The shoulder region was defined on the pressure map prior to each measurement for each participant by applying a very localized but large pressure on the map corresponding with the location of the following bony landmarks on both sides: the acromion to define the cranial point of the shoulder region; the inferior angle of the scapulae to define the bottom of the shoulders; and the lateral restrictions of the trunk to define the chest wall. Each participant was laid quietly prior to each measurement for $6 \mathrm{~min}$ in the respective position. ${ }^{6,17}$ Immediately after $6 \mathrm{~min}$, data were collected for $30 \mathrm{~s}$ for each of the seven positions. Areal pressure reflected the tallied pressure from all sensors. Peak pressure reflected the highest pressure from any one sensor. The median value for each of these measures was derived from the $30 \mathrm{~s}$ of collected data.

\section{Statistical methods}

Demographic data are presented as median (interquartile range) or frequency. The Friedman test was used to detect statistical differences between the seven positions for the measures of pressure and comfort. Differences were expressed as median differences and interquartile range. In case of statistical significance, the Wilcoxon test was used to localize the level of significance. The data on preferred position were analysed descriptively. For secondary analyses, Spearman's rank correlation coefficients were used to test for relations between pressure and comfort. Additionally, Spearman's rank correlations were performed to test whether pressure data need to be adjusted to body weight or body mass index. Statistical significance was set at alpha 0.05 . The Bonferroni correction was used to account for multiple statistical comparisons. All statistical analyses were performed using SPSS, version 20 (SPSS Inc., Chicago, IL, USA).

\section{RESULTS}

The characteristics of the 20 participants are shown in Table 1. Four were female and 16 were male. Median (interquartile range) age and time since injury were 36 years $(24-43)$ and 1 year (0.6-4), respectively. Neurological level ranged from C4-C7.

\section{Comfort}

The median VAS score for general comfort ranged from 6.4 to 9.4 for the seven positions whereas the corresponding score for shoulder 

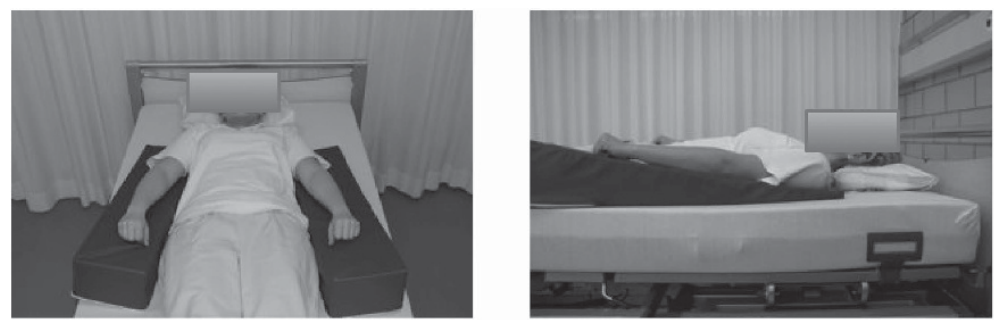

Position 1 (bed flat, shoulders in adduction and internal rotation)


Position 2 (top end of the bed inclined $30^{\circ}$, shoulders in adduction and internal rotation)

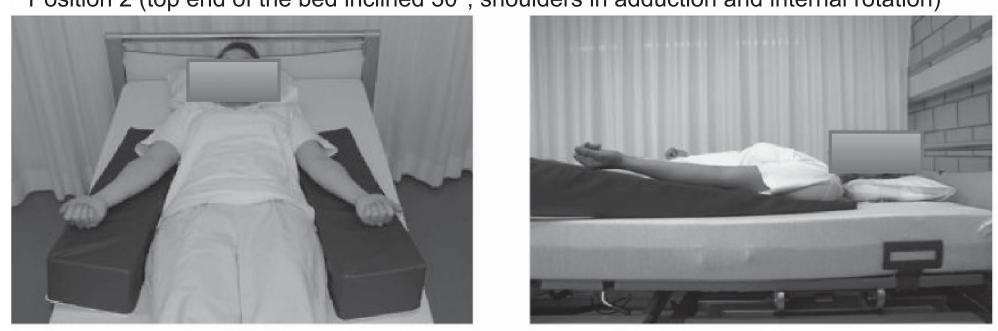

Position 3 (bed flat, shoulders in adduction and external rotation)
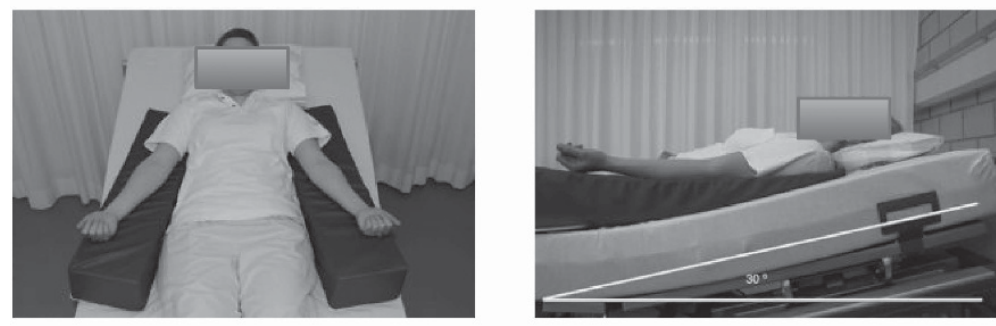

Position 4 (top end of the bed inclined $30^{\circ}$, shoulders in adduction and external rotation)



Position 5 (bed flat, shoulders in $45^{\circ}$ abduction and external rotation)



Position 6 (top end of the bed inclined $30^{\circ}$, shoulders in $45^{\circ}$ abduction and external rotation)

Figure 1. Description of the six standardized positions. 
Table 1 Characteristics of participants $(n=20)$

\begin{tabular}{lc}
\hline Male/female, $n$ & $16 / 4$ \\
Level of injury, $n$ & \\
C4 & 8 \\
C5 & 4 \\
C6 & 6 \\
C7 & 2 \\
AIS, $n$ & \\
A/B & $9 / 11$ \\
Time since injury (years) & $1(0.6-4.0)$ \\
Height (cm) & $177(173-184)$ \\
Weight (kg) & $71(62-78)$ \\
BMI (kg m ${ }^{-2}$ ) & $23(20-24)$ \\
Inpatient/outpatient, $n$ & $18 / 2$ \\
Nightly shoulder pain over preceding 4 weeks, NRS & $0(0-2.9)$ \\
\hline
\end{tabular}

Abbreviations: AIS, American Spinal Injury Association Impairment Scale; BMI, body mass index; NRS, numeric rating scale.

All data are reported as median (interquartile range) unless otherwise indicated.

Table 2 Shoulder pressure and comfort

\begin{tabular}{lcccc}
\hline Position & $\begin{array}{c}\text { Areal } \\
\text { pressure } \\
(\mathrm{mmHg})\end{array}$ & $\begin{array}{c}\text { Peak } \\
\text { pressure } \\
(\mathrm{mmHg})\end{array}$ & $\begin{array}{c}\text { General } \\
\text { comfort } \\
\text { (VAS) }\end{array}$ & $\begin{array}{c}\text { Shoulder } \\
\text { comfort } \\
\text { (VAS) }\end{array}$ \\
\hline 1 & $6.5(5.0-13.7)$ & $94.5(77.4-105.5)$ & $6.5(3.4-8.2)$ & $6.9(4.1-8.5)$ \\
2 & $6.2(4.8-9.5)$ & $93.5(71.9-139.6)$ & $7.1(5.4-8.5)$ & $7.7(4.7-8.8)$ \\
3 & $8.7(5.1-15.0)$ & $89.4(68.5-110.5)$ & $6.7(3.9-7.7)$ & $6.8(3.8-8.9)$ \\
4 & $6.6(4.8-13.3)$ & $94.5(74.1-120.9)$ & $6.7(3.9-7.8)$ & $6.1(4.4-8.0)$ \\
5 & $8.1(5.9-10.9)$ & $91.0(69.1-113.5)$ & $6.4(4.0-7.7)$ & $6.2(4.6-7.9)$ \\
6 & $6.4(4.8-11.9)$ & $91.5(70.1-119.2)$ & $6.6(4.9-7.8)$ & $6.9(3.6-8.6)$ \\
7 & $9.7(4.9-14.9)$ & $81.0(61.5-102.8)$ & $9.4(8.4-9.9)$ & $9.6(8.1-9.9)$
\end{tabular}

Abbreviation: VAS, visual analogue scale.

There was a statistical significance $(P<0.01)$ between position 7 and positions $1-6$ for general comfort and shoulder comfort.

Position 1: bed flat, shoulders in adduction and internal rotation.

Position 2: top end of the bed inclined $30^{\circ}$, shoulders in adduction and internal rotation.

Position 3: bed flat, shoulders in adduction and external rotation.

Position 4: top end of the bed inclined $30^{\circ}$, shoulders in adduction and external rotation.

Position 5: bed flat, shoulders in $45^{\circ}$ abduction and external rotation.

Position 6: top end of the bed inclined $30^{\circ}$, shoulders in $45^{\circ}$ abduction and external rotation.

Position 7: self-selected position.

Median (interquartile range) areal pressure, peak pressure, general comfort and shoulder comfort for the seven positions $(n=20)$.

comfort ranged from 6.1 to 9.6 with a score of 10 representing 'extremely comfortable'. Both general comfort and shoulder comfort were significantly higher for the self-selected position than any of the other six positions $(P<0.01)$ (see Table 2 for details). The median difference in general comfort between the self-selected position and the other six positions ranged from 1.8 to 3.1 on the VAS. The corresponding median difference in shoulder comfort between the self-selected position and the other six positions were similar ranging from 1.5 to 2.8 on the VAS (see Table 3 ).

\section{Preferred position}

The details of participants' preferred positions are provided in Table 4. Eighty percent (16/20) of participants preferred both arms close to the body (that is, both hands on the pelvis or the stomach), $15 \%(3 / 20)$ preferred an asymmetric arm position and 5\% (1/20) preferred both arms above the head. Most $(75 \% ; 15 / 20)$ selected a position, which placed both shoulders in an internally rotated position whereas the rest either selected a position, which placed both shoulders in an externally rotated position $(15 \% ; 3 / 20)$ or one
Table 3 Difference in general comfort and shoulder comfort

\begin{tabular}{lcc}
\hline Position & General comfort (VAS) & Shoulder comfort (VAS) \\
\hline $7-1$ & $2.7(1.1-5.4)$ & $2.3(1.0-4.3)$ \\
$7-2$ & $1.8(1.0-3.9)$ & $1.5(0.7-4.0)$ \\
$7-3$ & $2.5(1.1-5.1)$ & $1.7(0.5-5.5)$ \\
$7-4$ & $3.0(1.3-4.1)$ & $2.5(1.2-4.1)$ \\
$7-5$ & $3.1(1.4-5,1)$ & $2.8(0.7-4.1)$ \\
$7-6$ & $2.8(1.6-3.6)$ & $2.8(0.5-4.6)$ \\
\hline
\end{tabular}

Abbreviation: VAS, visual analogue scale.

Median difference (interquartile range) of VAS scores in general comfort and shoulder comfort between the self-selected position (position 7) and the other six positions (positions 1-6) $(n=20)$.

\section{Table 4 Preferred position}

\begin{tabular}{lc}
\hline Bed inclination & \\
Top end of the bed flat & $55 \%$ \\
Top end of the bed $10^{\circ}$ & $45 \%$ \\
Top end of the bed $30^{\circ}$ & $0 \%$ \\
& \\
Arm position & $15 \%$ \\
Asymmetrical & $85 \%$ \\
Symmetrical & \\
& \\
Glenohumeral rotation & $75 \%$ \\
Both arms in internal rotation & $15 \%$ \\
Both arms in external rotation & $10 \%$ \\
One arm in internal rotation, & \\
One arm in external rotation & \\
Glenohumeral abduction/adduction & \\
Glenohumeral abduction & $5 \% \%$ \\
Glenohumeral adduction & $5 \%$ \\
Wedges & \\
Two wedges & \\
One wedge & \\
No wedges & \\
\hline
\end{tabular}

The type of bed adjustment and arm position participants selected as their preferred position. The percentages refer to the percentage of participants who selected a particular bed adjustment or arm position $(n=20)$.

shoulder in each position $(10 \% ; 2 / 20)$. In addition, $80 \%(16 / 20)$ of participants preferred not to have wedges under the arms, $15 \%(3 / 20)$ preferred wedges under both arms and 5\% (1/20) preferred a wedge under one arm. Fifty-five percent (11/20) of participants preferred the bed flat whereas $45 \%(9 / 20)$ preferred the top end of the bed inclined with the most commonly selected inclination being $10^{\circ}$.

\section{Pressure}

The median of the peak pressures under the shoulders ranged from $81 \mathrm{mmHg}$ to $94.5 \mathrm{mmHg}$ for the seven positions (see Table 2) whereas the corresponding values for areal pressure ranged from $6.2 \mathrm{mmHg}$ to $9.7 \mathrm{mmHg}$. There was no statistical difference in peak pressure $(P=0.15)$ or areal pressure $(P=0.08)$ under the shoulders between the seven positions.

\section{Secondary analysis of correlations}

There was a weak correlation between comfort for the shoulders and peak pressure under the shoulders for three of the positions, namely position $4(R=0.45 ; P=0.03), 5(R=0.45 ; P=0.05)$ and 6 
$(R=0.48 ; P=0.03)$. However, neither areal pressure nor peak pressure was significantly correlated with body weight or body mass index for any of the seven positions.

\section{DISCUSSION}

Shoulder positioning programs for people with tetraplegia have been advocated for more than 40 years and are still widely used, yet no one to the best of our knowledge has systematically investigated the effect of different positions on patient comfort and pressure or on patients' preferences. These are three important factors, which should be considered when recommending specific positioning programs. The results of this study indicate that the position of the shoulder has little effect on pressure but a notable effect on comfort. The results also indicate that patients prefer to lie with their arms beside their bodies.

The position most participants elected as their preferred position was with the shoulders adducted and internally rotated and the hands either by their sides or on their stomachs. Only one participant elected a position with both shoulders abducted and the arms away from the body in a crucifix-type position even though this position is widely advocated by healthcare professionals. The preference for arms tucked in beside the body reflects how most people with or without a disability chose to sleep. This is presumably because this position is more comfortable and perhaps safer with less chance of arms being inadvertently knocked by others at night. The preference for sleeping in this position makes it imperative for researchers to provide clear evidence of the benefits of sleeping with the arms abducted in a crucifix position. If there is not a clear benefit then patients should be left to sleep and rest with their arms beside their body or as they chose.

Most participants preferred the top of the bed inclined only $10^{\circ}$, although $30^{\circ}$ or $45^{\circ}$ is commonly recommended. ${ }^{18,19}$ The main justification for inclining the bed $30^{\circ}$ or $45^{\circ}$ is to optimize respiratory function ${ }^{19}$ and reduce the risk of ventilatory-associated pneumonia, ${ }^{18}$ although these benefits have not been clearly demonstrated in people with SCI. Future studies need to clearly establish these benefits to justify this ongoing practice given patients prefer to sleep with less inclination than commonly recommended and the upright position exposes the patient to the risk of pressure ulcers ${ }^{7}$ and reduces vital capacity. $^{20}$

Comfort was not strongly associated with pressure, although there was a positive and significant correlation between shoulder comfort and peak pressure for some positions (position 4: $R=0.45, P=0.03$; position 5: $R=0.45, P=0.05$; position $6: R=0.48, P=0.03$ ). Interestingly, areal pressures were highest and peak pressures were lowest in the self-selected positions. It is not possible to draw too much from these findings, especially as there were no notable differences in peak- or areal pressures between the different positions. It would, however, appear that pressure is not strongly associated with comfort.

The main limitation of this study was that only the immediate effects of a short period of lying in each position were examined. In addition, the sample was small and not necessarily reflective of the population. Future studies could investigate the effects of lying in each position over an extended period of time and in a larger and more representative sample of people with tetraplegia. The scope could be also extended to investigate health-related issues by utilizing outcomes reflecting peripheral perfusion or pulmonary function.

\section{CONCLUSION}

The position of the shoulders has little effect on pressure but a notable effect on comfort. Patients prefer to sleep and lie with their arms beside their bodies, not with their arms in a crucifix position as commonly advocated. Researchers therefore need to provide clear evidence that placing the shoulders in a crucifix position or any variation on this is effective to justify this ongoing practice.

\section{DATA ARCHIVING}

There were no data to deposit.

\section{CONFLICT OF INTEREST}

The authors declare no conflict of interest.

\section{ACKNOWLEDGEMENTS}

The financial support of the Swiss Paraplegic Centre Nottwil.

1 Simpson LA, Eng JJ, Hsieh JTC, Wolfe DL, Spinal Cord Injury Rehabilitation Evidence Scire Research Team. The health and life priorities of individuals with spinal cord injury: a systematic review. J Neurotrauma 2012; 29: 1548-1555.

2 Curtis KA, Drysdale GA, Lanza RD, Kolber M, Vitolo RS, West R. Shoulder pain in wheelchair users with tetraplegia and paraplegia. Arch Phys Med Rehabil 1999; 18: $1-5$.

3 Eriks-Hoogland IE, de Groot S, Post MWM, van der Woude LH. Passive shoulder range of motion impairment in spinal cord injury during and one year after rehabilitation. J Rehabil Med 2009; 41: 438-444.

4 Crowe J, MacKay-Lyons M, Morris H. A multi-centre, randomized controlled trial of the effectiveness of positioning on quadriplegic shoulder pain. Physiother Can 2000; 52 $1-8$.

5 Glaesener JJJ, Denker MM, Petersen WW. Positioning techniques for the prevention of shoulder pain in the early phases of tetraplegia. Dtsch Krankenpflegez 1991; 44: 781-784.

6 Scott JA, Donovan WH. The prevention of shoulder pain and contracture in the acute tetraplegia patient. Paraplegia 1981; 19: 313-319.

7 Consortium for Spinal Cord Medicine Clinical Practice Guidelines. Pressure ulcer prevention and treatment following spinal cord injury: a clinical practice guideline for health-care professionals. J Spinal Cord Med 2001, pp S40-S101.

8 Harvey LA, Herbert RD. Muscle stretching for treatment and prevention of contracture in people with spinal cord injury. Spinal Cord 2002; 40: 1.

9 Ada L, Goddard E, McCully J, Stavrinos T, Bampton J. Thirty minutes of positioning reduces the development of shoulder external rotation contracture after stroke: a randomized controlled trial. Arch Phys Med Rehabil 2005; 86: 230-234.

10 Katalinic OM, Harvey LA, Herbert RD. Effectiveness of stretch for the treatment and prevention of contractures in people with neurological conditions: a systematic review. Phys Ther 2011; 91: 11-24.

11 Gebruers N, Truijen S, Engelborghs S, De Deyn PP. Is activity loss predictive for development of upper limb oedema after stroke? J Rehabil Med 2011; 43: 398-403.

12 Harvey L, Baillie R, Ritchie B, Simpson D, Pironello D, Glinsky J. Does three months of nightly splinting reduce the extensibility of the flexor pollicis longus muscle in people with tetraplegia? Physiother Res Int 2007; 12: 5-13.

13 Herbert RD, Balnave RJ. The effect of position of immobilisation on resting length, resting stiffness, and weight of the soleus muscle of the rabbit. J Orthop Res 1993; 11: 358-366.

14 Harvey LA, Glinsky JA, Katalinic OM, Ben M. Contracture management for people with spinal cord injuries. NeuroRehabilitation 2011; 28: 17-20.

15 Dietz V, Sinkjaer T. Spasticity. Handb Clin Neurol 2011; 109: 197-211.

16 Kirshblum SC, Waring W, Biering-Sorensen F, Burns SP, Johansen M, Schmidt-Read M et al. Reference for the 2011 revision of the international standards for neurological classification of spinal cord injury. J Spinal Cord Med 201134: 547-554.

17 Stinson M, Porter A, Eakin P. Measuring interface pressure: a laboratory-based investigation into the effects of repositioning and sitting. Am J Occup Ther 2002 56: 185-190.

18 Min YiP, Yuxin Leng P, Yu Bai MD, Gaiqi Yao P, Xi Zhu P. The evaluation of the effect of body positioning on intra-abdominal pressure measurement and the effect of intraabdominal pressure at different body positioning on organ function and prognosis in critically ill patients. J Crit Care 2012; 27: 222.e1-222.e6.

19 Griffiths H, Gallimore D. Positioning critically ill patients in hospital. Nurs Stand 2005; 19: 56-66.

20 Chen CF, Lien IN, Wu MC. Respiratory function in patients with spinal cord injuries: effects of posture. Paraplegia 1990; 28: 81-86. 